\title{
Relación entre las tasas de desempleo y los indicadores de absentismo por incapacidad temporal durante periodos de crisis económica
}

\author{
J.C. López Lópeza , M. Ballesteros Polo ${ }^{a}$, M. Sampere Valero ${ }^{a}$, R. Sacristán Nieto ${ }^{a}$, \\ S. Alguacil Garcia ${ }^{a}$, A. Ruiz Julian ${ }^{a}$, E. Santos Avila ${ }^{a}$, \\ S. García Rubia ${ }^{a}$, B. López Costa ${ }^{a}$, \\ J.M. Martinez Martinez $z^{a}$ \\ DOI: 10.12961/aprl.2018.21.02.3 \\ Recibido: 11 de diciembre de 2017 \\ Aceptado: 22 de febrero de 2018
}

\section{RESUMEN}

Introducción: Estudios realizados en varios países presentan una relación inversa entre las tasas de desempleo (TD) y los indicadores de absentismo por incapacidad temporal (IT) durante periodos de crisis económica.

Objetivo: Evaluar la relación entre indicadores de absentismo y el desempleo en España (2009-2015).

Métodos: Se obtuvieron tasas de incidencia (TI) y absentismo (TA) por IT de origen común (ITcc) a partir de datos de la Seguridad Social, así como las TD según datos del Instituto Nacional de Estadística. Se describió gráficamente la evolución temporal de los indicadores de ITcc y el desempleo en el periodo 2009-2015. Para explorar dicha relación también se realizaron gráficos de dispersión de los indicadores frente a las TD. Posteriormente, se ajustaron modelos de regresión lineal simple.

Resultados: Las TI y TA descienden en 2009-2013 (TI 2009=28,07\%, TI 2013=20,41\%, TA 2009=2,53\%, TA 2013=1,86\%) momento en que cambia su tendencia, iniciando una fase ascendente hasta 2015 (TI 2015=22,52\%, TA 2015=2,12\%). La TD asciende en 2009-2013 (TD 2009=17,86\%, TD 2013=26,10\%), pasando a descender hasta 2015 (TD 2015=22,06\%). Las TD y los indicadores de absentismo presentan una relación lineal inversa.

Conclusiones: Se observó una relación inversa entre el desempleo y los indicadores de absentismo en España. Son necesarios estudios específicos para poder evaluar hipótesis explicativas como las asociadas a los efectos de disciplina, selección y/o cambios en la fuerza de trabajo.

PALABRAS CLAVE: Incapacidad Temporal; Desempleo; Absentismo; Recesión Económica.

\section{THE RELATIONSHIP BETWEEN UNEMPLOYMENT RATES AND LOST WORK TIME DUE TO SICKNESS ABSENCE IN AN ECONOMIC RECESSION.}

\section{ABSTRACT}

Introduction: Different countries have shown an inverse relationship between unemployment rates (UR) and indicators of sickness absence during periods of economic recession.

Objective: To evaluate the relationship between indicators of sickness absence and unemployment in Spain during 2009-2015.

Methods: We obtained incidence and absenteeism rates of non-work related sickness absence (NWSA) from Social Security data, and UR from the National Institute of Statistics. The relationship between indicators of NWSA and UR in the period 2009 to 2015 was graphically described using time trend plots. Scatter plots of NWSA indicators were also made against UR. Finally, we fitted linear regression models.

Results: Incidence (IR) and absenteeism rates (AR) of NWSA showed downward trends in 2009-2013 (IR 2009=28.07\%, IR 2013=20.41\%; AR 2009=2.53\% and AR 2013=1.86\%), changing to an upward trend up to 2015 (IR 2015=22.52\%, AR 2015=2.12\%). Unemployment rates trended upward in 2009-2013 (UR 2009=17.86\%, UR 2013=26.10\%), and then changed to a downward trend through 2015 (UR 2015=22.06\%). There was an inverse relationship between UR and NWSA indicators.

Conclusions: As in previous international studies, we found an inverse relationship between unemployment rates and indicators of sickness absence in Spain. More detailed studies are needed to evaluate explanatory hypotheses, such as those associated with the effects of discipline, selection and/or changes in the workforce.

KEYWORDS: Sick Leave; Unemployment; Absenteeism; Economic Recession.

a. MC Mutual, Barcelona, España.
Correspondencia:

Juan Carlos López López

Departamento de Investigación y Análisis de Prestaciones, MC Mutual.

Provenza, 321. 08037 Barcelona

Tel. 934051244. Fax. 932553721

jlopez1@mc-mutual.com 


\section{INTRODUCCIÓN}

En el tercer trimestre del año 2007, la tasa de desempleo (TD) en España, del 7,93\%, era la más baja registrada desde los últimos 30 años del siglo XX. A partir de ese momento, y a consecuencia del inicio de una profunda crisis económica, la TD comenzó a subir, con un máximo en el primer trimestre de 2013, alcanzando al 26,94\% de la población activa ${ }^{1}$. En esos años, diversas organizaciones, como la Petita i Mitjana Empresa de Catalunya (PIMEC) ${ }^{2}$ o $\mathrm{ADECCO}^{3}$, se hacen eco de la existencia de cambios en los indicadores de absentismo de las empresas españolas.

Las publicaciones en otros países ponen de manifiesto la relación existente entre la TD y el absentismo laboral, entendiendo este como las ausencias al trabajo por motivos de salud. En el caso de las ausencias por contingencia común, trabajos como los de Askildsen ${ }^{4,5}$ y Dyrstad ${ }^{6}$ en Noruega, Fahr ${ }^{7}$ y Schon ${ }^{8}$ en Alemania, Arai ${ }^{9}$ en Suecia, Audas ${ }^{10}$ y Shoss ${ }^{11}$ en Estados Unidos o Scoppa $^{12}$ en Italia, señalan la existencia de una relación inversa entre desempleo y absentismo, de tal manera que cuando la TD aumenta, la tasa de absentismo disminuye.

En España, las referencias sobre este tema son muy escasas. Hay artículos centrados en el efecto del desempleo y el ciclo económico en las lesiones por accidentes de trabajo ${ }^{13}$, 14. En el caso del absentismo por contingencia común un estudio ${ }^{15}$ investigó los cambios en la duración de los episodios de IT considerando dos momentos, 2006 y 2010, antes y después del comienzo de la crisis. Sin embargo, según nuestro conocimiento no se han publicado investigaciones que analicen la relación entre el desempleo y el absentismo por incapacidad temporal de origen común (ITcc) en España. Evaluar la asociación entre el desempleo y el absentismo, puede permitir plantear hipótesis que permitan desarrollar estudios más dirigidos y específicos hacia los factores que expliquen la relación existente.

El objetivo de este trabajo es describir la relación entre los indicadores de absentismo por contingencia común y la tasa de desempleo en España en el periodo 2009-2015.

\section{MÉTODOS}

Diseño de estudio

Diseño ecológico de series temporales.

\section{Población}

Población trabajadora en España del periodo 2009-2015.

\section{Fuentes de información}

Los datos se obtuvieron a partir de información suministrada por la Seguridad Social ${ }^{16}$, seleccionándose los enlaces referentes a la población trabajadora por cuenta ajena y con la gestión de las contingencias comunes a cargo de las Mutuas Colaboradoras de la Seguridad Social. Las TD en el periodo 2009-2015 fueron obtenidas de Instituto Nacional de Estadística (INE)1.
Análisis estadístico

Indicadores de ITcc y desempleo

Los indicadores de ITcc que se utilizaron fueron la tasa de incidencia (TI) anual y la tasa de absentismo (TA) anual obtenidos para cada uno de los años en estudio (2009-2015). Estos se definen como:

TI anual $=\frac{\text { Número de casos que se inician en el año }}{\text { Población promedio en el año }}$

TA anual $=\frac{\text { Número de días naturales que transcurren en situación de ITcc }}{\text { Población promedio en el año x } 365 \text { días }}$

La TI anual hace referencia al número de procesos que se inician en el año correspondiente respecto al número de trabajadores-año. Por otro lado, la TA anual representa los días perdidos (días transcurridos en situación de ITcc) en el año correspondiente respecto al número de días naturales, del año.

Los datos obtenidos de la Seguridad Social proporcionaron información directa acerca de la incidencia media mensual por cada mil trabajadores protegidos (TI mensual) y la duración media de los procesos de IT con alta en el periodo (DM). Estos valores se convirtieron a los indicadores mediante los siguientes cálculos:

TI anual por $100=\frac{\text { TI mensual por } 1000 \times 12}{10}$

TA anual por $100=\frac{\text { TI anual por } 100 \times \text { DM }}{365 \text { días } \times 100}$

Relación entre absentismo y desempleo

Se describió gráficamente la evolución temporal de los indicadores de ITcc y la TD en el periodo 2009-2015. La relación entre los indicadores de ITcc y la TD se exploró en primer lugar mediante gráficos de dispersión. A partir de la relación observada se ajustaron los correspondientes modelos de regresión lineal, tomando como variables dependientes los indicadores de ITcc y como variable explicativa la TD. La relación se cuantificó mediante el coeficiente que acompañaba a la variable TD y el valor del $\mathrm{R}^{2}$. Los programas utilizados fueron Excel 2010 y R 3.3.1 $1^{17}$.

\section{RESULTADOS}

Las TI y TA por ITcc mostraron tendencias descendentes en 2009-2013, momento en que cambian sus tendencias, iniciando una fase ascendente hasta 2015. La TI en 2009 fue del 28,07\%, del 20,41\% en 2013 y del 22,52\% en el año 2015. La TA en 2009 fue del 2,53\%, del 1,86\% en 2013 y del 2,12\% en 2015 (Figura 1). Por otro lado, la TD presentó una evolución ascendente en el periodo 2009-2013, pasando de un $17,86 \%$ en 2009 a un $26,10 \%$ en 2013, para descender hasta un 22,06\% en 2015 (Figura 1). 


\section{FIGURA 1}

Tasas de incidencia, absentismo y desempleo.

España, 2009-2015.

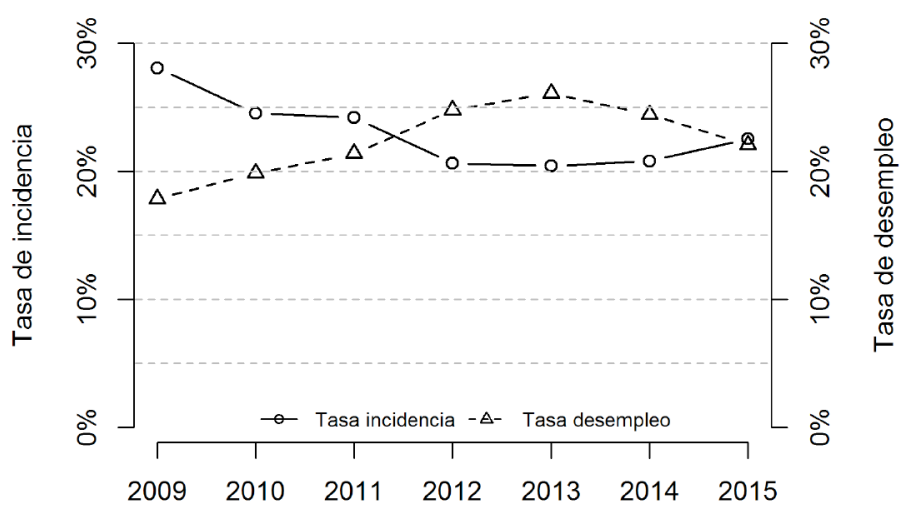

Año

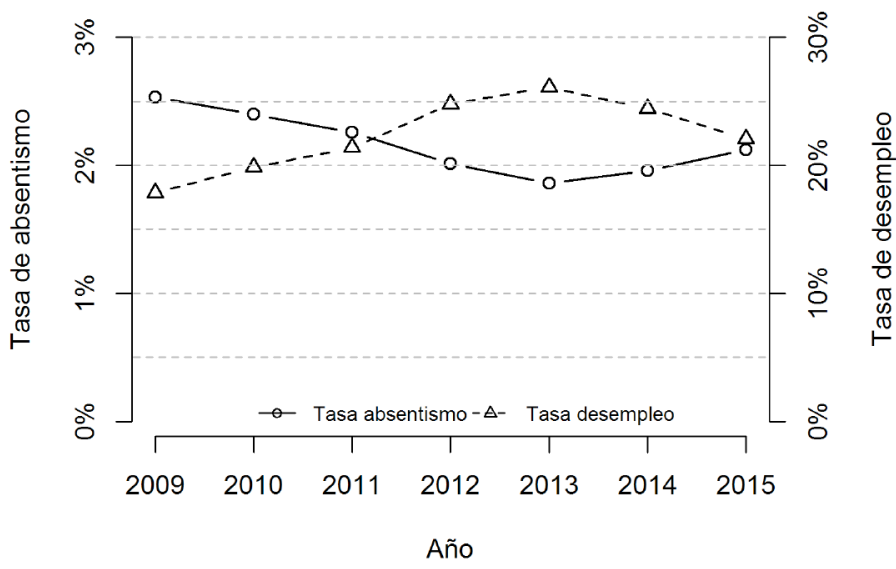

Finalmente, se observó una relación lineal inversa entre las TD y los indicadores de absentismo (Figura 2). Para la TI el modelo de regresión lineal ofrece un coeficiente para el desempleo de $-0,93(p<0,001)$ y un $R^{2}=93,7 \%$. Para la TA el coeficiente de desempleo se sitúa en $-0,08$ ( $p<0,001$ ) y se obtiene un $R^{2}=97,5 \%$.

\section{DISCUSIÓN}

Los resultados muestran una relación inversa entre el desempleo y el absentismo en España en el periodo 2009-2015. Es decir, cuando la TD mostraba una trayectoria ascendente (2009-2013), tanto la TI como la TA descendían cada año, y cuando en 2013 se produjo un punto de inflexión, y la TD comenzó a descender, los indicadores de ITcc empezaron a aumentar de valor.

Diferentes estudios ofrecen explicaciones a este hallazgo, pudiéndose mencionar tres modelos explicativos principales: el efecto de la disciplina, el efecto de la selección y el efecto de la carga de trabajo ${ }^{5,6,9}$.

El efecto de la disciplina (discipline effects) ${ }^{6,9}$, es el que mayor soporte recibe. Esta explicación propone que cuando el empleo escasea, las personas se esfuerzan en conservar el suyo, y por ello tratan de limitar las ausencias al trabajo, que podrían incrementar el riesgo de ser despedido en un momento de reducción de plantilla.
FIGURA 2

Relación entre la tasa de desempleo y la tasa de incidencia y absentismo. España, 2009-2015.

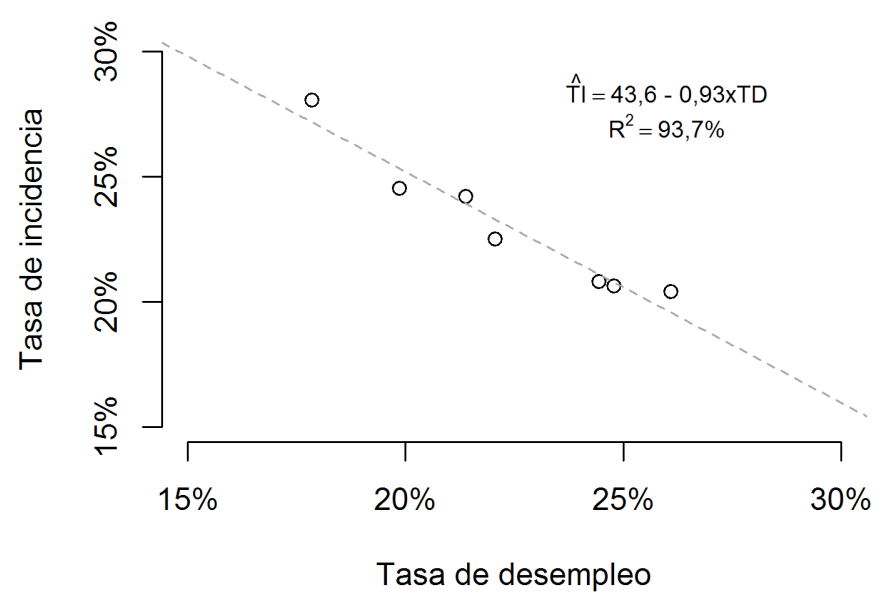

$\hat{\mathrm{TI}}=$ Tasa de incidencia estimada; $\mathrm{TD}=$ Tasa de desempleo.

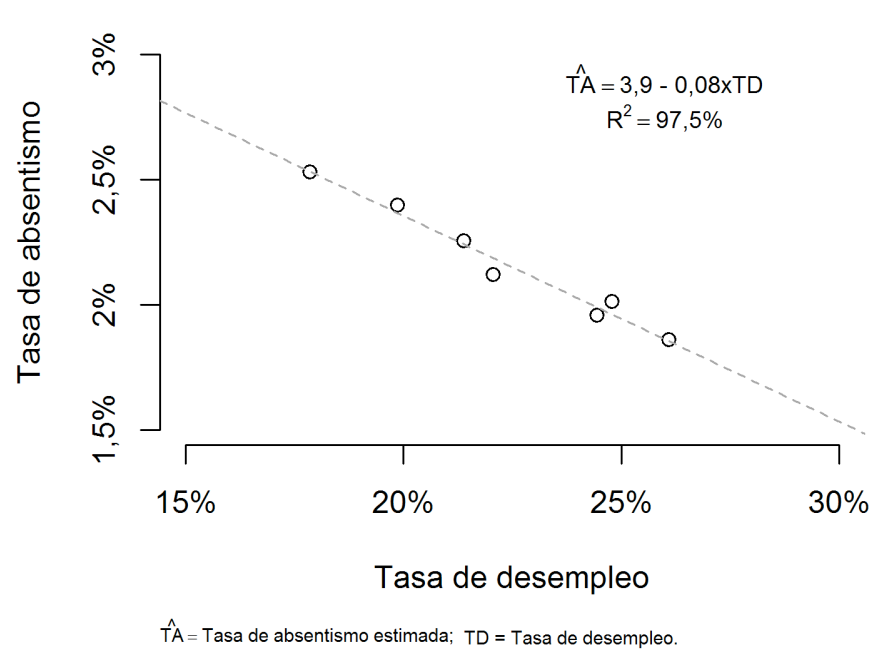

En este contexto, se puede llegar a una situación de presentismo ${ }^{18}$. La relación entre un historial de ausencias por enfermedad y un incremento de la probabilidad de ser despedido ha sido puesta de manifiesto por diferentes investigaciones ${ }^{19,20}$. Sin embargo, tal y como indica Leoni ${ }^{21}$, hay que considerar que la relación salud y desempleo es de doble dirección. Un efecto disciplina modificaría el comportamiento de los trabajadores, pero también expulsaría del empleo a los trabajadores con peor salud. Las evidencias sobre el efecto disciplina se basan en gran medida en los cambios en el comportamiento de los trabajadores que conservan el empleo a lo largo del ciclo económico, pero también en el diferente impacto según el nivel de protección de los trabajadores ${ }^{10,13,22}$.

El efecto de la selección ${ }^{6,9}$ (employer selection), o efecto de composición de la fuerza de trabajo (labour force composition) propone que en los momentos de recesión económica los trabajadores con peor salud tienen más riesgo de perder su trabajo, por lo que la población que permanece trabajando experimenta un incremento colectivo de su nivel de salud. Cuando cambia el ciclo, el reclutamiento de nuevos trabajadores incorpora, entre otras, personas que estaban en situación de desempleo por sus problemas de salud, y disminuye el nivel colectivo de salud. 
La teoría de la carga de trabajo ${ }^{5}$, menos mencionada, propone que la variación del absentismo está influenciada por la variación de la carga de trabajo. Cuando la economía entra en recesión, la carga de trabajo disminuye al tener las organizaciones más capacidad de producción que demanda. A medio plazo, las organizaciones terminan ajustando su plantilla a sus necesidades, disminuyendo de tamaño. Al cambiar el ciclo económico e incrementarse la demanda, esas organizaciones la afrontan con plantillas insuficientes en un primer momento, produciéndose un incremento de la carga de trabajo de sus empleados, lo cual a su vez tiene efectos negativos sobre su salud.

Como limitaciones del estudio se puede considerar que se ha seleccionado únicamente la población trabajadora afiliada al régimen general de la Seguridad Social y con la gestión de las contingencias comunes a cargo de las Mutuas Colaboradores de la Seguridad Social. Además, el desempleo corresponde a la encuesta de población activa y son datos de población total del sistema. Dado que el régimen general tiene unas características legales específicas en cuanto a la regulación de la ITcc, la generalización de nuestros hallazgos en el contexto y marco legal español se restringe a este régimen de afiliación, que es por otro lado el que acumula la mayoría de trabajadores en nuestro sistema de Seguridad Social. Según datos de la Seguridad Social, el porcentaje de trabajadores del régimen general en España se situó entre el 75 y el $80 \%$ en el periodo $2009-2015^{16}$. Otra limitación vendría determinada por el cálculo de los días perdidos (numerador de la tasa de absentismo) ya que se calculan a partir del producto de la tasa de incidencia y la duración media. Esto podría representar una infra o sobreestimación de la tasa de absentismo. A pesar de estas limitaciones, considerando el objetivo del estudio, y tal como se comenta al inicio de la discusión, la tendencia de la tasa de absentismo y su relación con la tasa de desempleo obtenida es la observada en otros países. Esto podría indicar que si existiera un sesgo en los indicadores, no afectaría en gran medida a la forma de la tendencia, aunque si pudiera afectar a la escala de la gráfica.

Por otro lado, los modelos ajustados obtenidos para cuantificar la relación entre los indicadores de ITcc y el desempleo son lineales. Sin embargo, es improbable que la relación entre el desempleo y los indicadores de ITcc sea lineal para valores excesivamente elevados de la tasa de desempleo, dado que eso implicaría la existencia, al menos teórica, de un absentismo cero si la tasa de desempleo fuera lo bastante elevada. Este hecho es señalado por Dyrstad $^{6}$, que propone una relación cóncava entre absentismo y desempleo, de tal manera que cuando comienza a incrementarse la tasa de desempleo, la respuesta de los indicadores de ITcc es al principio muy lenta, para posteriormente incrementarse su tasa de cambio respecto al desempleo.

Un aspecto que no ha podido ponerse de manifiesto es el carácter precíclico de los indicadores de ITcc respecto al desempleo destacado en otras investigaciones ${ }^{5,10,23}$, en las cuales se observa que los indicadores de ITcc cambian antes de que cambie el ciclo económico. La causa probable es la falta de detalle en las series temporales utilizadas, concretamente las referidas a los indicadores de ITcc, que son anuales. Es posible que utilizando series trimestrales pudiera también apreciarse este fenómeno en el caso de España.

Arch Prev Riesgos Labor 2018; 21 (2): 80-84
Como implicaciones prácticas de este estudio podemos destacar al menos dos. Por un lado, el uso de los resultados obtenidos permite una mejor comprensión de las causas que afectan a los indicadores de absentismo por ITcc. Específicamente, se muestra que la variación de los indicadores de una empresa pueden ser también debidos a factores externos (es decir, no únicamente a factores internos o propios) que aparecen debido al ciclo económico. Por consiguiente, estos factores afectarían de forma global a toda la población trabajadora. Por otro lado, la consistencia en la tendencia cíclica observada en el tiempo, permite prever la repetición de las tendencias observadas en el futuro. Esto permitiría realizar una mejor planificación y toma de medidas a nivel de recursos asistenciales y económicos en función de los resultados esperados.

En resumen, la relación inversa observada entre el desempleo y los indicadores de ITcc permite plantear diferentes hipótesis sobre los factores que puedan explicar dicha relación. Futuras investigaciones deberían estar dirigidas a establecer qué factores son los que contribuyen al patrón observado. Esto permitiría establecer políticas nacionales específicas en el mercado de trabajo y/o en las empresas.

\section{BIBLIOGRAFÍA}

1. Instituto nacional de estadística [página principal en Internet] [citado 9 Febrero 2018]. Disponible en http://www.ine.es.

2. Petita i Mitjana Empresa de Catalunya. Absentisme laboral en temps de crisi. 2011. [citado 9 Febrero 2018]. Disponible en: HYPERLINK "https://www. pimec.org” https://www.pimec.org /sites /default /files /documents_pagines / absentisme_laboral_en_temps_de_crisi.pdf

3. ADECCO. Informe ADECCO sobre el absentismo. Madrid 2012.

4. Askildsen J E, Bratberg E, Nilsen OA. Sickness absence over the Business Cycle. Department of Economics. University of Bergen. Working Papers in Economics No 0400. 2000. [citado 9 Febrero 2018]. Disponible en:http://www.uib. no/filearchive/04-2000_1.pdf.

5. Askildsen JE, Bratberg E, Nilsen OA. Unemployment, labor force composition and sickness absence: a panel data study. Health Econ. 2005;14:1087-101.

6. Dyrstad JM, Ose SO. Non-linear Unemployment Effects in Sickness Absence: Discipline or Composition Effects? Department of Economics. Norwegian University of Science and Technology. Working Paper No 25. 2002. [citado 9 Febrero 2018]. Disponible en: https://brage.bibsys.no/xmlui/handle/11250/267159. 7. Fahr R, Frick B. On the inverse relationship between unemployment and absenteeism: evidence from natural experiments and worker heterogeneity. IZA Discussión Paper No 3171. November 2007. [citado 9 Febrero 2018]. Disponible en: https://papers.ssrn.com/sol3/papers.cfm?abstract_id=1039221.

8. Schön M. Unemployment, Sick Leave and Health. 2015 [citado 9 Febrero 2018]. Disponible en: HYPERLINK "http://www.sole-jole.org/15406.pdf" http://www.sole-jole.org/15406.pdf.

9. Arai M, Thoursie PS. Incentives and selection in cyclical absenteeism. Labour Econ 2005; 12: 269-280.

10. Audas R, Goddard J. Absenteeism, seasonality, and the business cycle. Journal of Economics and Business. 2001; 534: 405-419.

11. Shoss MK, Penney LM. The economy and absenteeism: a macro-level study. J Appl Psychol. 2012;97:881-9.

12. Scoppa, V. and D. Vuri (2014). Absenteeism, unemployment and employment protection legislation: evidence from Italy. IZA Journal of Labor Economics. 2014; 3: 1-25.

13. de la Fuente VS, López MAC, González IF, Alcántara OJ, Ritzel DO. The impact of the economic crisis on occupational injuries. J Safety Res. 2014;48:77-85. 14. Fernández-Muñiz B, Montes-Peón JM, Vázquez-Ordás CJ. Occupational accidents and the economic cycle in Spain 1994-2014. Safety science. 2016. HYPERLINK “https://doi.org/10.1016/j.ssci.2016.02.029” https://doi.org/10.1016/j. ssci.2016.02.029. Disponible en: http://www.sciencedirect.com /science/article/pii/S0925753516300066.

15. Murcia López G, Delclòs Clanchet J, Ubalde López M, Calvo Bonacho E, Benavides FG. Has the Spanish economic crisis affected the duration of sickness absence episodes?. Social Science \& Medicine. 2016; 160, 29-34.

16. Seguridad Social. [página principal en Internet] [citado 9 Febrero 2018] Disponible en: HYPERLINK "http://www.seg-social.es/Internet_1/index.htm" 
http://www.seg-social.es/Internet_1/index.htm.

17. R Core Team. R: A language and environment for statistical computing. R Foundation for Statistical Computing, Vienna, Austria. 2016. URL [página principal en Internet] [citado 9 Febrero 2018]. Disponible en: https://www.Rproject.org/.

18. Gustafsson K, Marklund S. Consequences of sickness presence and sickness absence on health and work ability: A Swedish prospective cohort study. Int J Occup Med Environ Health. 2011; 24: 153-65.

19. Hesselius P. Does sickness absence increase the risk of unemployment? J Socio-economics. 2007; 36: 288-310.

20. Virtanen M, Kivimäki M, Vahtera J, Elovainio M, Sund R, et al. Sickness absence as a risk factor for job termination, unemployment, and disability pension among temporary and permanent employees. Occup Environ Med 2006;63:212-17.

21. Leoni T. Differences in sick leave between employed and unemployed workers: what do they tell us about the health dimension of unemployment? WIFO Working Papers No 372, Vienna 2010. [citado 9 Febrero 2018]. Disponible en: HYPERLINK "http://www.wifo.ac.at/publikationen?detail-view=yes\& publikation_id=39861" http://www.wifo.ac.at/publikationen?detail-view=yes\&publikation_id=39861.

22. Pfeifer C. Cyclical absenteeism among private sector, public sector and selfemployed workers. Health Economics 2013; 22: 366-70.

23. Pichler S. Sickness Absence, Moral Hazard, and the Business Cycle. Health Econ. 2015; 24:692-710.

\section{CONGRESO PRLINNOVACIÓN}

17-18 de abril de 2018, Madrid

\section{Información:}

Asociación para la Innovación en Prevención y Salud

PRLInnovación

Plaza Puerta del Sol, 5, 4 planta, 28013 Madrid, España

Tel.: 917692410

Web: http://congreso.prlinnovacion.com/

SIOP 2018

XXXIII CONFERENCIA ANUAL

DE LA SOCIEDAD DE PSICOLOGÍA INDUSTRIAL

Y DE LAS ORGANIZACIONES

19-21 de abril de 2018, Chicago (EE.UU.)

\section{Información:}

SIOP Administrative Office

440 E Poe Rd, Ste 101, Bowling Green,

$\mathrm{OH} 43402$, EE.UU

Tel.: 419-353-0032.

Fax: 419-352-2645

E-mail: SIOP@siop.org

Web: www.siop.org/conferences/18con/default.aspx

\section{ICOH 2018}

XXXII CONGRESO INTERNACIONAL

DE SALUD LABORAL

29 de abril-4 de mayo de 2018, Dublín (Irlanda)

Información:

Ciara Ryan

Conference Partners Ltd

Suite 11-13, The Hyde Building, The Park Carrickmines,

Dublin 18, Irlanda

Tel: + 35312166685

E-mail: icoh2018@conferencepartners.ie

Web: http://icoh2018.org/wp/

\section{CONGRESO INTERNACIONAL}

SOBRE SALUD Y SEGURIDAD EN EL TRABAJO

6-9 de mayo de 2018, Estambul (Turquía)

\section{Información:}

Scientific Secretariat

Inönü Bulvarı No:42 Pk: 06520 Emek/Ankara, Turquía

E-mail: eto.isggm@csgb.gov.tr

Web: http://tioshconference.gov.tr/ 